\title{
Chapter 24 \\ On a Possible Phase Transition Between \\ Hadron Matter and Quark-Gluon Matter: 1981
}

\author{
Rolf Hagedorn
}

\begin{abstract}
We employ the technique of the analytically continued grand canonical pressure partition function to show that, under physically meaningful boundary conditions (non-existence of external confining vessels, i.e., no fixed volumes), the energy density and similar intensive quantities do indeed have, in a statistical bootstrap model of extended hadrons (van der Waals type volume corrections), the singularity claimed in previous papers. Earlier results obtained with an entirely different technique (which had been criticized) are recovered and shown to be correct. The technique used here is useful in all cases where the volume is not imposed from the outside but results from the internal dynamics of the system, as is generally the case in high energy physics and astrophysics.
\end{abstract}

\subsection{Introduction}

Hadrons have finite sizes and consist internally of quarks, antiquarks, and gluons, though none of these constituents has ever been observed as a free particle. They seem to be confined to the inside of hadrons.

Consider a hadron gas at temperature $T$. At low $T$, it behaves more or less as an ideal gas, if $T$ increases to the order of $\lesssim 100 \mathrm{MeV}$, pion creation sets in and if $T$ is increased further, heavier resonances and baryon-antibaryon pairs are produced. When $T$ becomes sufficiently large, particle production becomes so strong that the energy density of the 'gas' reaches the value of the internal energy density of its

Preprint CERN-TH-3207 dated 30 November 1981, unpublished; See also the expanded version Preprint CERN-TH-3392 dated 18 August 1982 with title "The Pressure Ensemble as a Tool for Describing the Hadron-Quark Phase Transition” published in Z. Phys. C 17, pp. 265-281 (1983). With kind permission of (C Springer Science+Business Media, 1983.

R. Hagedorn: (deceased) CERN-TH, 1211 Geneve 23, Switzerland

J. Rafelski $(\square)$

Department of Physics, The University of Arizona, Tucson, AZ 85721, USA

(C) The Author(s) 2016

J. Rafelski (ed.), Melting Hadrons, Boiling Quarks - From Hagedorn Temperature

to Ultra-Relativistic Heavy-Ion Collisions at CERN,

DOI 10.1007/978-3-319-17545-4_24 
constituents. In other words, the hadrons (having finite volume) begin to overlap and finally might form one single hadron. At that stage we have no longer to do with a hadron gas, but with an interacting quark-gluon gas. Increasing the temperature further would lead to a free quark-gluon gas confined to a macroscopic hadron [1].

The following question arises: is this transition from a hadron gas to a quarkgluon gas a smooth transition (like ionization) or a phase transition? There are two approaches to this problem: experimental and theoretical. Experimental facts suggest a phase transition: mean transverse momenta of particles produced in high energy hadron collisions may be interpreted in terms of a temperature which, with increasing collision energy, rapidly reaches a limit of the order of $160 \mathrm{MeV}$ [2] (the exact value is difficult to determine because many secondary effects arising from the spacetime history of a collision disturb the ideal picture of a phase transition at a certain critical temperature). Limiting temperatures indicate phase transitions.

The theoretical approach is suffering from lack of a theory. We only have models. There is a choice of models describing the hadron side and another choice of models for the quark-gluon side, but no analytical model which contains both [3]. A single, closed and consistent analytical model unifying both aspects would be ideal. If we had one which described hadronic as well as quark-gluon systems, we could use it to find theoretical support for either a phase transition or for a smooth transition. A phase transition would be indicated by a singularity (pole, branch point) in the grand canonical partition function at some real temperature, while a smooth transition would require the absence of singularities on the real $T$ axis.

In this situation, the general habit is to take some hadron gas (or nuclear matter) model and some quark-gluon model and try to fit them together. This procedure leads then to two different partition functions, one for low $T$ (hadron side) and one for high $T$ (quark-gluon side). If the two pressure curves thus obtained cross at some temperature, it is often claimed that a phase transition has thereby been established and located. This is unjustified, as one easily sees from a counterexample: a dilute hydrogen gas might, according to this philosophy, be described as an ideal gas of $2 N$ protons plus $2 N$ electrons. The pressure curves do cross, but we know that, in this system, there is no phase transition; instead a smooth shift of the chemical equilibrium between molecules, atoms, ions, and electrons takes place when the temperature changes.

Only the explicit exhibition of a singularity (in at least one of the two models to be fitted together) proves that the model under consideration has a phase transition (in the vicinity of the singularity).

The statistical bootstrap model of hadronic matter in its most recent form has been claimed to have a singular curve in the $\mu, T$ diagram, along which the energy density is constant and equal to the bag energy density, i.e., the energy density of the hadronic clusters constituting the gas, while the pressure vanishes there [4]. Taking this singularity as indicating a phase transition to a quark-gluon phase [5] seems most natural; the more so as the description of the other side, in terms of a free quark-gluon gas with perturbative corrections [6], leads to vanishing pressure and to the usual bag energy density in the same $\mu, T$ region where the hadron critical curve lies. 
The model of [4] is a statistical bootstrap model with baryon number conservation and proper volumes of the constituent hadrons and hadron clusters. These proper volumes grow in proportion to the cluster mass. As the strong interaction is in this model represented by all possible particle reactions (hadron chemistry), the number of particles is not conserved and, in calculating the partition function, summed from 0 to infinity. The mass spectrum (listing all possible hadrons and clusters) turns out to be exponentially increasing with the cluster mass. It is this exponential increase which generates the singularity via an integration over masses up to infinity.

In a recent paper [7], the results of [4] were criticized on the basis of the following argument: if particle volumes grow in proportion to the mass, the mass integration is necessarily cut off when the sum of all particle volumes reaches the externally given volume $V$. Likewise the sum over particle numbers is cut off. Thus, trivially, no singularity can occur. What is not trivial is that, as the authors show, the thermodynamic limit

$$
\lim _{V \rightarrow \infty} \frac{1}{V} \ln Z(\beta, V)
$$

exists for all $\beta$. Hence, even in this limit, still no singularity exists in spite of the exponential spectrum and in spite of the fact that now integrations and sums do go to infinity. This proof does not, however, apply to the situation under which the singularity was found. In [4], the limit was not taken in the usual way, viz., as in Eq. (24.1): first calculate $\ln Z$ for fixed $V$, then let $V \rightarrow \infty$. Instead, the 'available volume' $\Delta=V-\sum V_{i}$, where $V_{i}$ is the proper volume of the $i$ th particle, was used as a volume parameter and kept constant. Thus $V=\Delta+\sum V_{i}$, so that, when sums over particle numbers and integrals over masses were done, $V$ was pushed to infinity. Then expectation values $\langle V(\beta, \Delta, \lambda)\rangle,\langle E(\beta, \Delta, \lambda)\rangle$, etc., could be calculated and densities could be defined by $\langle E(\beta, \Delta, \lambda)\rangle /\langle V(\beta, \Delta, \lambda)\rangle$, etc., which did indeed show a singularity. Since therefore the existence of a singularity depends on the limiting procedure, it seems important to clear this up.

A simple example shows that there is nothing like a universally 'correct' limiting procedure, but that different limiting procedures correspond to different physical situations. Imagine a high pressure container of volume $V$ filled completely with water at room temperature and atmospheric pressure and then hermetically closed. One may heat it up to any temperature and the water will not boil; putting infinitely many such boxes together and removing interior walls $(V \rightarrow \infty)$ will change nothing. If, on the other hand, one closes the vessel by a movable piston, one sees the water boil if pressure and temperature fall in a certain interval. In this last case, the water pushes the volume to ever larger values similarly to the situation considered in [4].

We believe that at temperatures and densities where hadron matter changes into quark-gluon matter, no fixed volumes should be used in theoretical considerations, since boxes do not exist in this regime. Forces keeping a system together (the tendency to cluster is just such an internal force, while gravity might be considered as an external one) control pressure and densities rather than volume. 
The method of the 'available volume' $\Delta$ used in [4] seems therefore to be adapted to reality. Nevertheless, one may argue that even such a $\Delta$ cannot be controlled and therefore should not be used as an external variable.

In this paper, the results of [4] will be rederived in a different way which does not make use of a volume-like variable. The technical tool is the grand canonical pressure partition function.

Our units are $\hbar=c=k=1$, mass unit $\mathrm{MeV}$, metric $(1,-1,-1,-1)$. Notation is as in [4].

\subsection{The Grand Canonical Pressure Partition Function}

\section{Introduction}

Given the grand canonical partition function $Z(\beta, V, \lambda)$, where $\beta=1 / T, V$ a fixed external volume, and $\lambda=\exp (\mu / T)$ a fugacity ensuring the conservation of some charge-type quantum number $Q$, the grand canonical pressure partition function $\Pi(\beta, \xi, \lambda)$ is defined by [8]

$$
\Pi(\beta, \xi, \lambda):=\int_{0}^{\infty} \mathrm{d} V \mathrm{e}^{-\xi V} Z(\beta, V, \lambda),
$$

where $\xi$ is a new, intensive parameter related to the volume in a similar way as $\beta$ is related to the energy and $\mu$ to some conserved quantity. The larger $\xi$, the stronger is the exponential volume suppression in the integral of Eq. (24.2). Thus $\xi$ is a measure for the pressure and hence the name of this partition function.

Rewriting Eq. (24.2),

$$
\Pi(\beta, \xi, \lambda)=\int_{0}^{\infty} \mathrm{d} V \mathrm{e}^{-V[\xi-\ln Z(\beta, V, \lambda) / V]},
$$

we can read off for which values of $\xi$ the integral converges, provided that the thermodynamic limit

$$
\lim _{V \rightarrow \infty} \frac{1}{V} \ln Z(\beta, V, \lambda)
$$

exists:

$$
\xi>\xi_{0}(\beta, \lambda):=\lim _{V \rightarrow \infty}[\ln Z(\beta, V, \lambda) / V] \equiv \beta P(\beta, \lambda)
$$

where $P$ is the pressure. 
We define a function $g(\beta, V, \lambda)$ as the difference between $\ln Z / V$ and its limit:

$$
g(\beta, V, \lambda):=\xi_{0}(\beta, \lambda)-\frac{1}{V} \ln Z(\beta, V, \lambda)
$$

It can be shown under very general conditions [8, item (d)] that, if the thermodynamic limit exists, this limit commutes with the differential operators $\partial / \partial \beta$ and $\partial / \partial \lambda$. We assume these conditions to be fulfilled. Thus,

$$
\left.\begin{array}{l}
\lim _{V \rightarrow \infty} g(\beta, V, \lambda) \\
\lim _{V \rightarrow \infty} \frac{\partial g}{\partial \beta}(\beta, V, \lambda) \\
\lim _{V \rightarrow \infty} \frac{\partial g}{\partial \lambda}(\beta, V, \lambda)
\end{array}\right\}=0 .
$$

$\Pi(\beta, \xi, \lambda)$ has a singularity at $\xi_{0}(\beta, \lambda)$. Its nature (pole, branch point) depends on $g(\beta, V, \lambda)$. In principle, it is possible to continue the analytic function $\Pi(\beta, \xi, \lambda)$, defined by the integral representation in Eq. (24.2) for $\operatorname{Re} \xi>\xi_{0}$, into the whole complex plane beyond the convergence domain of the integral. Therefore it might well be possible that quantities derived from $\Pi(\beta, \xi, \lambda)$ have a physical meaning for $\xi$ values where the integral representation of $\Pi(\beta, \xi, \lambda)$ does not exist.

That this is indeed the case and that the singularity at $\xi_{0}$ is absent in meaningful physical quantities will now be shown. It implies that the singularity at $\xi_{0}$ has nothing to do with a phase transition, in contradistinction to singularities of $Z(\beta, V, \lambda)$.

It is convenient to define a new function whose limit is $\Pi(\beta, \xi, \lambda)$ :

$$
\begin{array}{r}
\Pi_{W}(\beta, \xi, \lambda):=\int_{0}^{W} \mathrm{~d} V \mathrm{e}^{-\xi V} Z(\beta, V, \lambda) \\
=\int_{0}^{W} \mathrm{~d} V \mathrm{e}^{-V\left(\xi-\xi_{0}\right)-V g(\beta, V, \lambda)}, \\
\lim _{W \rightarrow \infty} \Pi_{W}(\beta, \xi, \lambda)=\Pi(\beta, \xi, \lambda) .
\end{array}
$$

We now calculate the energy density. First we define the expectation value of the total energy:

$$
\left\langle E_{W}(\beta, \xi, \lambda)\right\rangle:=-\frac{1}{\Pi_{W}} \frac{\partial \Pi_{W}}{\partial \beta}=\frac{\int_{0}^{W} \mathrm{~d} V \mathrm{e}^{-\xi V} Z(\beta, V, \lambda)\langle E(\beta, \xi, \lambda)\rangle}{\int_{0}^{W} \mathrm{~d} V \mathrm{e}^{-\xi V} Z(\beta, V, \lambda)},
$$


where $\langle E(\beta, \xi, \lambda)\rangle=-\partial \ln Z(\beta, V, \lambda) / \partial \beta$ was used. Similarly, we define the expectation value of the volume:

$$
\left\langle V_{W}(\beta, \xi, \lambda)\right\rangle:=-\frac{1}{\Pi_{W}} \frac{\partial \Pi_{W}}{\partial \xi}=\frac{\int_{0}^{W} \mathrm{~d} V \mathrm{e}^{-\xi V} V Z(\beta, V, \lambda)}{\int_{0}^{W} \mathrm{~d} V \mathrm{e}^{-\xi V} Z(\beta, V, \lambda)}
$$

The energy density is then

$$
\mathscr{E}(\beta, \xi, \lambda):=\lim _{W \rightarrow \infty} \frac{\left\langle E_{W}(\beta, \xi, \lambda)\right\rangle}{\left\langle V_{W}(\beta, \xi, \lambda)\right\rangle}
$$

Similarly, if $Q$ is the quantum number conserved by $\lambda$, the quantum number density $q(\beta, \xi, \lambda)$ is

$$
q(\beta, \xi, \lambda):=\lim _{W \rightarrow \infty} \frac{\left\langle Q_{W}(\beta, \xi, \lambda)\right\rangle}{\left\langle V_{W}(\beta, \xi, \lambda)\right\rangle}, \quad\left\langle Q_{W}(\beta, \xi, \lambda)\right\rangle=-\frac{1}{\Pi_{W}} \lambda \frac{\partial \Pi_{W}}{\partial \lambda}
$$

In this formalism, the usual thermodynamic limit is replaced by the limit $W \rightarrow \infty$.

We now use the explicit form given by the last member of Eq. (24.7) to calculate $\mathscr{E}(\beta, \xi, \lambda)$ :

$$
\begin{gathered}
\mathscr{E}(\beta, \xi, \lambda)=\lim _{W \rightarrow \infty} \frac{\partial \Pi_{W} / \partial \beta}{\partial \Pi_{W} / \partial \xi}, \\
\frac{\partial \Pi_{W}}{\partial \beta}=\int_{0}^{W} V\left(\frac{\partial \xi_{0}}{\partial \beta}-\frac{\partial g}{\partial \beta}\right) \mathrm{e}^{-V\left(\xi-\xi_{0}\right)-V g} \mathrm{~d} V \\
\frac{\partial \Pi_{W}}{\partial \xi}=-\int_{0}^{W} V \mathrm{e}^{-V\left(\xi-\xi_{0}\right)-V g} \mathrm{~d} V
\end{gathered}
$$

Since $\xi_{0}$ is independent of $V$, we have

$$
\mathscr{E}(\beta, \xi, \lambda)=-\frac{\partial \xi_{0}(\beta, \lambda)}{\partial \beta}+\lim _{W \rightarrow \infty} \frac{\int_{0}^{W} \mathrm{e}^{-V\left(\xi-\xi_{0}\right)-V g} V \frac{\partial g}{\partial \beta} \mathrm{d} V}{\int_{0}^{W} \mathrm{e}^{-V\left(\xi-\xi_{0}\right)-V g} V \mathrm{~d} V} .
$$

We now recall Eq. (24.4), viz., $\xi_{0}=\beta P$. Hence, if the second term of Eq. (24.13) were absent, we would recover the usual thermodynamic limit definition

$$
\mathscr{E}=-\frac{\partial(\beta P)}{\partial \beta}=-\frac{\partial}{\partial \beta}\left[\lim _{V \rightarrow \infty} \frac{\ln Z(\beta, V, \lambda)}{V}\right] .
$$


Next we observe that, for $\xi>\xi_{0}$, the integrals in Eq. (24.13) converge in the limit so that, unless $g(\beta, V, \lambda)=0$, the second term is a non-vanishing function $C(\beta, \xi, \lambda)$. Indeed, $\xi>\xi_{0}$ implies exponential suppression of large volumes. Therefore this function $C(\beta, \xi, \lambda)$ represents the corrections to the energy density coming from finite volume effects.

If finite volume effects are neglected already in defining $Z(\beta, V, \lambda)$, then $g \equiv 0$. In that case $\Pi=1 /\left(\xi-\xi_{0}\right)$ has a simple pole at $\xi_{0}$ which cancels out in $\mathscr{E}(\beta, \xi, \lambda)$ and the second term of Eq. (24.13) is absent. Furthermore, both $\mathscr{E}(\beta, \xi, \lambda)$ and $q(\beta, \xi, \lambda)$ are trivial analytic functions of $\xi$, namely, constants in the whole $\xi$ plane. This particularly simple case is a good illustration of what happens. While $\langle E\rangle$ and $\langle V\rangle$ both have a pole at $\xi_{0}$ and become negative at $\xi<\xi_{0}$, the energy density does not care: the pole cancels and with it the whole $\xi$ dependence. The $\mathscr{E}$ calculated from Eq. (24.13) is just the usual one obtained from $-\partial[\ln Z / V] / \partial \beta$.

In the more general case where finite volume effects are not neglected, i.e., $g(\beta, V, \lambda) \neq Q$, the correction term in Eq. (24.13) is present for $\xi>\xi_{0}$. It vanishes, however, identically for $\xi \leq \xi_{0}$ due to our assumption that $\lim _{V \rightarrow \infty} \partial g / \partial \beta=0$ [see Eq. (24.6)]. The simple proof is by de l'Hôpital's rule. Thus, if finite volume effects are included in the definition of $Z(\beta, V, \lambda)$, we recover the usual thermodynamic limit results for $\mathscr{E}(\beta, \xi, \lambda)$ for all $\xi \leq \xi_{0}$ [there $\mathscr{E}(\beta, \xi, \lambda)$ becomes independent of $\xi$ ], while for $\xi>\xi_{0}$, finite volume corrections appear explicitly. All this is physically obvious: for $\xi<\xi_{0}$, large volumes have an exponentially increasing weight in the integration, whence the main contributions come from 'infinite' volumes where finite volume effects are absent by definition. Once this happens, it does not matter how fast the exponential weight increases. Therefore, $\mathscr{E}(\beta, \lambda)$ is independent of $\xi$ for $\xi \leq \xi_{0}$. Again, $\mathscr{E}(\beta, \xi, \lambda)$ defined by Eq. (24.13) is a meaningful physical quantity which may be evaluated at any $\xi$, while the individual integrals in Eq. (24.13) go to infinity in the limit $W \rightarrow \infty$.

This introduction thus results in two useful conclusions:

- Whatever the singularity of $\Pi(\beta, \xi, \lambda)$ at $\xi_{0}$ may be, it has no significance for quantities like $\mathscr{E}(\beta, \xi, \lambda)$ and $q(\beta, \xi, \lambda)$. While $\langle\mathscr{E}(\beta, \xi, \lambda)\rangle$ and $\langle V(\beta, \xi, \lambda)\rangle$ do have a singularity at $\xi_{0}$ and may become meaningless for $\xi \leq \xi_{0}$, the singularity (pole, branch point, cut) cancels in calculating the above densities, which may be evaluated at any $\xi$.

- If one wishes to obtain explicit finite volume corrections, one must evaluate densities at $\xi>\xi_{0}$. If, on the other hand, one evaluates at $\xi \leq \xi_{0}$, it is irrelevant whether or not finite volume terms, or more precisely, surface terms, have been included in the definition of $Z(\beta, V, \lambda)$ : they are suppressed by the exponentially increasing weight of large volumes.

The real power of the pressure partition function formalism is this: it may happen that $\Pi(\beta, \xi, \lambda)$ can be calculated explicitly as an analytic function of $\xi$, while the direct analytic calculation of $Z(\beta, V, \lambda)$ is impossible. In that case, we can obtain exact results from $\Pi(\beta, \xi, \lambda)$ which we could not obtain from $Z(\beta, V, \lambda)$. This is precisely what happens in our problem of the van der Waals statistical bootstrap model. 
In applying the technique introduced here to our explicit problem, the situation will be slightly different from the above, already fairly general case. However, our main conclusion that $\mathscr{E}(\beta, \xi, \lambda)$ and $q(\beta, \xi, \lambda)$ can be continued beyond the singularity $\xi_{0}$ remains valid. As this will be seen explicitly, we kept this additional complication out of our discussion above.

\section{How Shall We Use $\Pi(\beta, \xi, \lambda)$ ?}

Having decided that the usual thermodynamic limit-which requires, at least in a gedanken experiment, the existence of rigid boxes with a fixed volume-does not correspond to the situation where hadron matter goes over to quark matter, we shall not evaluate $\Pi(\beta, \xi, \lambda)$ at $\xi_{0}(\beta, \lambda)$, but rather consider $\xi$ as an independent thermodynamic variable on the same footing as $\beta$ and $\lambda$.

As the only relevant quantities to be calculated are densities which, as we have seen, ignore the existence of the singularity at $\xi_{0}$, we adopt the following philosophy. If we can obtain an analytic expression for $\Pi(\beta, \xi, \lambda)$, then we proceed to calculate from it expressions for the interesting densities which then are also analytic functions (not containing the singularity at $\xi_{0}$ ). We consider these functions as analytic continuations of the functions defined via the integral representation of Eq. (24.2) beyond the region of convergence of the latter. We then evaluate these functions at a $\xi$ value appropriate to the physical situation.

Before we turn to the application to our specific problem, we have to generalize Eq. (24.2) relativistically, since it will be used with a relativistic formulation of $Z(\beta, V, \lambda)$. In this formulation, $V$ is a timelike four-vector, so $\xi$ must also be written as a timelike four-vector. Hence the generalization of Eq. (24.2) is

$$
\Pi(\beta, \xi, \lambda)=\int \frac{\mathrm{d} V_{\mu} \xi^{\mu}}{\sqrt{\xi_{\mu} \xi^{\mu}}} \mathrm{e}^{-\xi_{\mu} V^{\mu}} Z(\beta, V, \lambda)
$$

where $Z(\beta, V, \lambda)$ is already a Lorentz invariant. The integration in Eq. (24.14) goes over the forward cone $V^{0} \geq 0$. Going to the rest frame of $\xi$ leads back to Eq. (24.2).

\subsection{The Hadron Gas}

\section{Introduction}

The grand canonical partition function of the strongly interacting hadron gas described by statistical bootstrap is written [4]

$$
Z(\beta, V, \lambda)=\sum_{N=0}^{\infty} \frac{1}{N !} \int \prod_{i=1}^{N}\left[\frac{2(V-\mathscr{A} p)_{\mu} p_{i}^{\mu}}{(2 \pi)^{3}}\right]_{+} \tau\left(p_{i}^{2}, \lambda\right) \mathrm{e}^{-\beta_{\mu} p_{i}^{\mu}} \mathrm{d}^{4} p_{i} .
$$


Here $V^{\mu}$ is the external volume (to be integrated away), $\mathscr{A} p^{\mu}$ is $\mathscr{A}=1 / 4 \mathscr{B}$ times the sum of all four-momenta $\sum p_{i}^{\mu}$, with $4 \mathscr{B}$ being the bag energy density [9], $\tau\left(p^{2}, \lambda\right)$ is the hadron cluster mass spectrum with baryon number conservation as follows from the bootstrap equation, and $\beta^{\mu}$ is the inverse temperature four-vector. The whole expression is written as a Lorentz invariant, following Touschek [10].

Attractive forces are represented by the mass spectrum, repulsive forces by the van der Waals type correction to the volume: from the total volume, the proper volumes of all particles are subtracted ${ }^{1}$ :

$$
V^{\mu}-\mathscr{A} p^{\mu}=V^{\mu}-\mathscr{A} \sum_{i=1}^{N} p_{i}^{\mu}=V^{\mu}-\sum_{i=1}^{N} V_{i}^{\mu},
$$

which is the covariant generalization of what would be $V-\mathscr{A} \sum m_{i}$ in a nonrelativistic formulation. That $\mathscr{A} p_{i}^{\mu}$ is the proper four-volume of the $i$ th particle is a byproduct obtained in formulating the bootstrap equation [11]. It agrees (in the particle's rest frame) with the nuclear physics, where the volume is proportional to the mass, and with the bag model [9]. For further information, see [4] and references therein.

The subscript + on the square bracket in Eq. (24.15) indicates that each single bracket is to be $\geq 0$. This is guaranteed if

$$
\begin{aligned}
& p_{i}^{2}>0,(V-\mathscr{A} p)^{2} \geq 0, \\
& p_{i}^{0}>0,(V-\mathscr{A} p)^{0} \geq 0 .
\end{aligned}
$$

The first two are trivial requirements since we are dealing with physical particles. The last two ensure positivity. Implicitly, they define the limits of the sum over $N$ and of the integrations over $p_{i}$.

It is this van der Waals correction in Eq. (24.16) which prevents the integrations in Eq. (24.15) from factorizing into $N$ independent integrals and which, moreover, makes the boundary of the sum and the integrals so complicated that it seems hopeless to calculate $Z(\beta, V, \lambda)$ without using drastic approximations. Introducing the pressure partition function $\Pi(\beta, \xi, \lambda)$ is not only suggested by the physical situation (no boxes), but it also solves the technical problem just mentioned.

\section{Digression: The Pointlike Hadron Gas}

For later use, we need to consider the pointlike case. If no volume correction is applied in Eq. (24.15), the integrations factorize. Moreover the sum and integrals

\footnotetext{
${ }^{1}$ The factor of 4 multiplying the proper volumes of the constituents in the usual van der Waals correction is omitted, since it is specific to a gas of identical hard spheres, while here the clusters are deformable and of different sizes.
} 
are unrestricted. We introduce under the integrals the identity

$$
\tau\left(p_{i}^{2}, \lambda\right) \equiv \int \delta_{0}\left(p_{i}^{2}-m_{i}^{2}\right) \tau\left(m_{i}^{2}, \lambda\right) \mathrm{d} m_{i}^{2}
$$

The $N$ identical integrals, evaluated in the common rest frame of the volume $\left[V^{\mu}=\right.$ $(V, 0,0,0)]$ and of the thermometer $\left[\beta^{\mu}=(1 / T, 0,0,0)\right]$, can then be summed up over $N$ and yield an exponential function, so that

$$
\frac{1}{V} \ln Z_{\mathrm{pt}}(\beta, V, \lambda):=\int_{0}^{\infty} \tau\left(m^{2}, \lambda\right) \mathrm{e}^{-\beta \sqrt{p^{2}+m^{2}}} \mathrm{~d} m^{2} \frac{p^{2} \mathrm{~d} p}{2 \pi^{2}},
$$

where $Z_{\mathrm{pt}}$ is the 'point particle partition function'. Note that the expression in Eq. (24.19) is independent of the volume $V$. The remaining integral is, in the Statistical Bootstrap Model, simply related to the 'bootstrap function' $\phi(\beta, \lambda)$ $[4,11]$ :

$$
\frac{1}{V} \ln Z_{\mathrm{pt}}(\beta, V, \lambda)=-\frac{2}{H(2 \pi)^{3}} \frac{\partial \phi(\beta, \lambda)}{\partial \beta}=: f(\beta, \lambda),
$$

where $f(\beta, \lambda)$ is a shorthand notation for later use. The function $\phi(\beta, \lambda)$ is analytically and numerically well known [12] and easy to compute. Thus $f(\beta, \lambda)$ may be considered as a known function.

The function $\phi(\beta, \lambda)$ has, for given $\lambda$, a square root singularity at some $\beta^{*}(\lambda)$, namely $[4,12]$

$$
\phi(\beta, \lambda) \underset{\beta \rightarrow \beta^{*}}{\longrightarrow} \ln 2-h(\lambda) \sqrt{\beta-\beta^{*}(\lambda)} .
$$

The curve $\beta^{*}(\lambda)$ would thus be a singular curve of the point particle model. We shall see that it will also be a singular curve of the model with nonzero particle volumes. From Eqs. (24.20) and (24.21), we infer

$$
\ln Z_{\mathrm{pt}}(\beta, \lambda) \underset{\beta \rightarrow \beta^{*}}{\longrightarrow} \sim \frac{1}{\sqrt{\beta-\beta^{*}}} \longrightarrow \infty
$$

Consequently, we obtain for the energy density

$$
\mathscr{E}_{\mathrm{pt}}(\beta, \lambda)=-\frac{1}{V} \frac{\partial \ln Z_{\mathrm{pt}}}{\partial \beta} \underset{\beta \rightarrow \beta^{*}}{\longrightarrow} \sim \frac{1}{\sqrt{\beta-\beta^{*}}},
$$

for the baryon number density

$$
v_{\mathrm{pt}}(\beta, \lambda)=\frac{1}{V} \lambda \frac{\partial \ln Z_{\mathrm{pt}}}{\partial \lambda} \underset{\beta \rightarrow \beta^{*}}{\longrightarrow} \sim \frac{1}{\sqrt{\beta-\beta^{*}}},
$$


and for the pressure

$$
P_{\mathrm{pt}}(\beta, \lambda)=\frac{1}{\beta V} \ln Z_{\mathrm{pt}} \underset{\beta \rightarrow \beta^{*}}{\longrightarrow} \sim \frac{1}{\sqrt{\beta-\beta^{*}}} .
$$

\section{The Real Hadron Gas}

The pressure partition function of the real hadron gas is given by the definition (24.14) with $Z(\beta, V, \lambda)$ taken from Eq. (24.15):

$$
\Pi(\beta, \xi, \lambda)=\int \frac{\mathrm{d} V_{\mu} \xi^{\mu}}{\sqrt{\xi_{\mu} \xi^{\mu}}} \mathrm{e}^{-\xi_{\mu} V^{\mu}} \sum_{N=0}^{\infty} \frac{1}{N !} \int \prod_{i=1}^{N}\left[\frac{2(V-\mathscr{A} p)_{\mu} p_{i}^{\mu}}{(2 \pi)^{3}}\right]_{+} \tau\left(p_{i}^{2}, \lambda\right) \mathrm{e}^{-\beta_{\mu} p_{i}^{\mu}} \mathrm{d}^{4} p_{i}
$$

We make the change of variables

$$
(V-\mathscr{A} p)^{\mu}=: x^{\mu}
$$

Positivity then requires [see Eq. (24.17)]

$$
x_{\mu} x^{\mu} \geq 0, \quad x^{0} \geq 0
$$

With this substitution and with the identity in Eq. (24.18), we obtain

$$
\Pi(\beta, \xi, \lambda)=\int \frac{\mathrm{d} x_{\mu} \xi^{\mu}}{\sqrt{\xi_{\mu} \xi^{\mu}}} \mathrm{e}^{-\xi_{\mu} x^{\mu}} \sum_{N=0}^{\infty} \frac{1}{N !} \int \prod_{i=1}^{N} \frac{2 x_{\mu} p_{i}^{\mu}}{(2 \pi)^{3}} \mathrm{e}^{-(\beta+\mathscr{A} \xi)_{\mu} p_{i}^{\mu}} \tau\left(m_{i}^{2}, \lambda\right) \mathrm{d} m_{i}^{2} \frac{\mathrm{d}^{3} p_{i}}{2 p_{0 i}}
$$

where $p^{\mu}=\sum p_{i}^{\mu}$ has been used. Now the positivity condition is automatically satisfied by integrating over the forward light cone of $x$. The explicit $p$ dependence due to the volume correction in Eq. (24.15) has disappeared from the volume factors $x_{\mu} p_{i}^{\mu}$ and is shifted as $\xi_{\mu} p_{i}^{\mu}$ to the exponent where it factorizes. Thus the integrals over the $p_{i}$ are again all identical and unrestricted, as if we had a pointlike gas.

We assume the temperature to be measured in the rest frame of the expectation value of the volume $\left\langle V^{\mu}\right\rangle$, whence $\beta \| \xi$. As $\Pi(\beta, \xi, \lambda)$ is a Lorentz scalar, we evaluate in the common rest frame of $\beta$ and $\xi$.

One of the $N$ identical integrals in Eq. (24.29) is then ${ }^{2}$

$$
\int \frac{2\left(x_{0} p^{0}-\mathbf{x} \cdot \mathbf{p}\right)}{(2 \pi)^{3}} \mathrm{e}^{-(\beta+\mathscr{A} \xi) \sqrt{p^{2}+m^{2}}} \tau\left(m^{2}, \lambda\right) \mathrm{d} m^{2} \frac{\mathrm{d}^{3} p}{2 \sqrt{p^{2}+m^{2}}},
$$

${ }^{2}$ From now on, we write $\beta:=\sqrt{\beta_{\mu} \beta^{\mu}}$ and $\xi:=\sqrt{\xi_{\mu} \xi^{\mu}}$. 
where $\mathbf{x} \cdot \mathbf{p}$ vanishes upon angular integration, so that, with $x:=x^{0}$, the integral reduces to [see Eqs. (24.19) and (24.20)]

$$
x \int \tau\left(m^{2}, \lambda\right) \mathrm{e}^{-(\beta+\mathscr{A} \xi) \sqrt{p^{2}+m^{2}}} \mathrm{~d} m^{2} \frac{p^{2} \mathrm{~d} p}{2 \pi^{2}}=x f(\beta+\xi \mathscr{A}, \lambda) .
$$

Thus Eq. (24.29) becomes

$$
\Pi(\beta, \xi, \lambda)=\sum_{N=0}^{\infty} \frac{1}{N !} \int_{0}^{\infty} \mathrm{e}^{-\xi x} x^{N} \mathrm{~d} x[f(\beta+\xi \mathscr{A}, \lambda)]^{N} .
$$

The $x$ integration yields $N ! / \xi^{N+1}$, so that finally,

$$
\Pi(\beta, \xi, \lambda)=\frac{1}{[\xi-f(\beta+\xi \mathscr{A}, \lambda)]},
$$

where $f(\beta+\xi \mathscr{A}, \lambda)$ is the point particle expression of Eq. (24.19) taken at $\beta+\xi \mathscr{A}$. As mentioned earlier, $f(x, y)$ is a perfectly known function, both numerically and analytically. Thus, within the framework of the Statistical Bootstrap Model with extended particles, we have obtained a simple analytical expression for $\Pi(\beta, \xi, \lambda)$ defined in the whole $\xi$ plane [the difference from the case discussed in Sect. 24.2 is that $\xi$ appears in $f(\beta+\xi \mathscr{A}, \lambda)]$.

\section{Interpretation}

\section{The Usual Thermodynamic Limit with Fixed $V \rightarrow \infty$}

From Eq. (24.4), we know that the singular point $\xi_{0}(\beta, \lambda)$ of $\Pi(\beta, \xi, \lambda)$ is equal to $\beta P(\beta, \lambda)$ with the usual thermodynamic limit prescription

$$
\begin{gathered}
\beta P_{(V)}=\xi_{0}=\lim _{V \rightarrow \infty} \frac{1}{V} \ln Z(\beta, V, \lambda), \\
\xi_{0}(\beta, \lambda)=\text { root of equation } \xi=f(\beta+\mathscr{A} \xi, \lambda) .
\end{gathered}
$$

Finding this root is a simple numerical exercise, which we shall not execute here since we are not interested in this pressure, which is irrelevant for our physical problem.

We can now give a simple proof (without using any approximations) of the statement [7] that, in the usual thermodynamic limit procedure, the singularity at $\beta^{*}(\lambda)$ cannot be reached by any $\beta>0$, or in other words, that $\lim _{V \rightarrow \infty} \ln Z(\beta, V, \lambda) / V$ has no singularity on the real $T$ axis. We must show only that $\xi_{0}(\beta, \lambda)$ has no singularity. 
Equation (24.34) states that

$$
\xi_{0}(\beta, \lambda)=f\left(\beta+\mathscr{A} \xi_{0}(\beta, \lambda), \lambda\right)
$$

The singularity $f(x, \lambda) \sim\left[x-\beta^{*}(\lambda)\right]^{-1 / 2}$ [see Eq. (24.22)] cannot be reached. Assume that indeed $x=\beta+\mathscr{A} \xi_{0}(\beta, \lambda) \rightarrow \beta^{*}(\lambda)$. Then by Eq. (24.35), $\xi_{0} \rightarrow \infty$ and $\beta+\mathscr{A} \xi_{0} \rightarrow \infty$, contradicting the assumption. This holds for all $\beta>0$. Hence $\lim _{V \rightarrow \infty} \ln Z(\beta, V, \lambda) / V$ is analytic along the whole $T$ axis, together with all its derivatives.

Having already decided that in our problem the usual thermodynamic limit does not correspond to reality, we do not pursue this line further.

\section{Hot Hadron Matter: No Fixed Volume}

According to our philosophy stated in Sect. 24.2, we now evaluate $\Pi(\beta, \xi, \lambda)$ and its derivatives at fixed $\beta, \xi, \lambda$. Applying the definitions of Sect. 24.2 to Eq. (24.33), we immediately find

$$
\begin{aligned}
& \mathscr{E}(\beta, \xi, \lambda)=\frac{\langle E\rangle}{\langle V\rangle}=\frac{\mathscr{E}_{\mathrm{pt}}(\beta+\mathscr{A} \xi, \lambda)}{1+\mathscr{A} \mathscr{E}_{\mathrm{pt}}(\beta+\mathscr{A} \xi, \lambda)}, \\
& v(\beta, \xi, \lambda)=\frac{\langle b\rangle}{\langle V\rangle}=\frac{v_{\mathrm{pt}}(\beta+\mathscr{A} \xi, \lambda)}{1+\mathscr{A} \mathscr{E}_{\mathrm{pt}}(\beta+\mathscr{A} \xi, \lambda)},
\end{aligned}
$$

where, bearing in mind Eq. (24.20),

$$
\begin{gathered}
\mathscr{E}_{\mathrm{pt}}(\beta+\mathscr{A} \xi, \lambda)=-\left.\frac{\partial}{\partial \beta^{\prime}} f\left(\beta^{\prime}, \lambda\right)\right|_{\beta^{\prime}=\beta+\mathscr{A} \xi}, \\
\nu_{\mathrm{pt}}(\beta+\mathscr{A} \xi, \lambda)=\lambda \frac{\partial}{\partial \lambda} f(\beta+\mathscr{A} \xi, \lambda) .
\end{gathered}
$$

The energy density and baryon number density no longer contain the pole at $\xi_{0}$. They are analytic functions of $\beta, \xi, \lambda$ for all real values

$$
1 \leq \lambda<\infty, \quad \beta+\xi \mathscr{A}>\beta^{*}(\lambda)
$$

As $\xi$ is now an independent variable [and no longer related to $\beta$ and $\lambda$ by an equation like (24.35)], the singularity $\beta+\xi \mathscr{A}=\beta^{*}(\lambda)$ can be reached. There $\mathscr{E}_{\mathrm{pt}}$ and $v_{\mathrm{pt}}$ go to infinity [see Eqs. (24.23) and (24.24)] and thus $\mathscr{E}(\beta, \xi, \lambda)_{\text {crit }}=1 / \mathscr{A}$, while $v(\beta, \xi, \lambda)_{\text {crit }} \neq 0, \infty$.

As any $\xi>0$ corresponds to an external force trying to compress the system [see Eq. (24.2)], we consider $\xi=0$ to be the appropriate value for a system which determines its own volume dynamically. Thus for the hadron gas without external 
forces

$$
\begin{aligned}
& \mathscr{E}(\beta, \lambda)=\frac{\mathscr{E}_{\mathrm{pt}}(\beta, \lambda)}{1+\mathscr{A} \mathscr{E}_{\mathrm{pt}}(\beta, \lambda)}, \\
& v(\beta, \lambda)=\frac{v_{\mathrm{pt}}(\beta, \lambda)}{1+\mathscr{A} \mathscr{E}_{\mathrm{pt}}(\beta, \lambda)},
\end{aligned}
$$

which are the results already derived in [4] with the 'available volume' technique.

In astrophysical applications, $\xi$ can be used to take gravitational pressure into account. This has the effect of replacing the singular curve $\beta^{*}(\lambda)$ by a singular surface $\beta^{*}(\lambda, \xi)$. The limiting values of $\mathscr{E}$ and $v$ on this critical surface can then be calculated. For $\mathscr{E}$, it is again $1 / \mathscr{A}=$ const., as seen from Eq. (24.36).

A small conceptual problem arises with the pressure. We have stated often enough that the usual definition (24.34) is useless here. We must therefore define the pressure as in kinetic gas theory: there it is the result of the stochastic cannonade of the wall of the vessel by the gas particles. The pressure is found to be proportional to the average normal component of the momenta of the particles hitting the wall. Here, where we do not have material walls, we may define the pressure as being proportional to the average normal momentum component of particles passing through an imaginary surface from left to right. Then, going through the usual textbook derivation, we find that

$$
\beta P=\frac{\langle N\rangle}{\langle V\rangle},
$$

where all the dynamics is hidden in $\langle N\rangle$, the average number of clusters, and $\langle V\rangle$, the volume chosen by the system. One should not expect here a van der Waals type of equation, because there $N$ and $V$ are fixed external parameters, so that the ideal gas equation has to be corrected. Here this is not necessary. In the second paper of [4], Eq. (24.43) was indeed derived in the 'available volume' formalism. Here we take it as the definition of the pressure.

It remains to calculate $\langle N(\beta, \xi, \lambda)\rangle$. In Eq. (24.32), we multiply $f$ by a fugacity $\eta$ and obtain, instead of Eq. (24.33),

$$
\Pi(\beta, \xi, \lambda, \eta):=\frac{1}{\xi-\eta f(\beta+\mathscr{A} \xi, \lambda)} .
$$

Obviously,

$$
\langle N(\beta, \xi, \lambda)\rangle=\left.\frac{\eta}{\Pi} \frac{\partial \Pi(\beta, \xi, \lambda, \eta)}{\partial \eta}\right|_{\eta=1}
$$


Thus, with Eq. (24.20),

$$
\frac{\langle N\rangle}{\langle V\rangle}=\left.\frac{\eta f(\beta+\mathscr{A} \xi, \lambda)}{1+\eta A \mathscr{E}_{\mathrm{pt}}(\beta+\mathscr{A} \xi, \lambda)}\right|_{\xi=0, \eta=1}=\frac{\beta P_{\mathrm{pt}}(\beta, \lambda)}{1+\mathscr{A} \mathscr{E}_{\mathrm{pt}}(\beta, \lambda)} .
$$

Hence, with Eq. (24.43),

$$
P(\beta, \lambda)=\frac{P_{\mathrm{pt}}(\beta, \lambda)}{1+\mathscr{A} \mathscr{E}_{\mathrm{pt}}(\beta, \lambda)}
$$

which is the same as in [4].

From Eqs. (24.23) and (24.25), we see that $\mathscr{E}_{\mathrm{pt}}$ diverges more strongly than $P_{\mathrm{pt}}$ on the critical curve. Thus $P(\beta, \lambda)_{\text {crit }}=0$. This is not surprising, since on the critical curve our 'gas' has coalesced into one single 'particle' of infinite volume which does not move. In a complete model unifying the hadron side and the quark side, the pressure should not go to zero. In our case, where two different models have to be fitted together at the singularity, the pressure on the quark-gluon side rises steeply. The usual Maxwell construction then gives a region of constant vapour pressure along an isotherm $[6$, item $(d)]$.

\subsection{Conclusions}

It has been shown how the thermodynamic limit procedure must be adapted to the real physical situation. Different procedures may give different results: one may exhibit a singularity while the other does not, and yet both are correct-they simply apply to different physical boundary conditions. In the particular problem of the transition from hadron to quark-gluon matter, the usual grand canonical partition function does not lead to a singularity. We consider it (in the context of extended particles and a van der Waals type volume correction) as badly suited to describe our problem, because it assumes that a rigid volume containing the system can exist. At the transition from hadron matter to quark matter, this assumption is principally wrong. In an earlier attempt to do better [4], we introduced the 'available volume' as a new, independent variable in place of the volume. The result was that the thus modified grand canonical partition function had a singularity indicating a phase transition. In the present paper, we have confirmed the results of [4] using the grand canonical pressure partition function, which seems to be tailored to our specific problem. It can be stated that, in the Statistical Bootstrap Model with extended hadrons (volume proportional to mass), a phase transition does occur as claimed earlier [4] and that the objections raised in [7], though correct in themselves, do not apply to physical reality in the temperature and density regime considered here.

Furthermore, we do not accept the conclusions of a recent paper [13], namely that it is important which singularity of $\Pi(\beta, \xi, \lambda)$ is reached first, the (trivial) one at $\xi_{0}(\beta, \lambda)$ or some other at $\beta^{*}(\lambda)$ originating from $Z(\beta, V, \lambda)$. These conclusions 
disregard the disappearance of the singularity at $\xi_{0}$ from densities. (We do not claim, however, that there might not be cases to which the analysis presented in [13] is relevant.)

Acknowledgements I am grateful to J. Rafelski, H. Satz, and L. Van Hove for extensive discussions and constructive criticism (this does not imply that they would agree on everything said here).

Open Access This book is distributed under the terms of the Creative Commons Attribution Noncommercial License which permits any noncommercial use, distribution, and reproduction in any medium, provided the original author(s) and sources are credited.

\section{References}

1. J. Baacke, Acta Phys. Pol. B 8, 625 (1977)

2. This fact is known already to cosmic ray physicists and has been stated in so many papers that it is impossible to list them all. Let two more recent ones represent the whole lot:

(a) A.T. Laasanen, C. Ezell, L.J. Gutay, W.N. Schreiner, P. Schübelin, L. von Lindern, F. Turkot, Phys. Rev. Lett. 38, 1 (1977);

(b) G.W. van Appeldoorn, S. Barshay, D. Harting, D.J. Holthuizen, P.G. Kuijer, B.J. Pijlgroms, V. Karimäki, R. Kinnunen, M. Korkea-Aho, P. Johnson, D. Michaelides, Ch. Michaelidou, D.G. Patel, Z. Physik C, Part. Fields 7, 235 (1981)

3. We do not consider here Monte Carlo lattice calculations which may indeed cover both sides and do suggest a phase transition, but which cannot exhibit singularities in the strict sense. See, e.g., J. Engels, F. Karsch, I. Montvay, H. Satz, Phys. Lett. B 102, 332 (1981) and references therein

4. (a) R. Hagedorn, J. Rafelski, Phys. Lett. B 97, 136 (1980);

(b) R. Hagedorn, J. Rafelski, From hadron gas to quark matter I, in Statistical Mechanics of Quarks and Hadrons, ed. by H. Satz (North Holland, Amsterdam, 1981)

5. N. Cabibbo, G. Parisi, Phys. Lett. B 59, 67 (1974)

6. (a) J.I. Kapusta, Nucl. Phys. B 148, 461 (1979);

(b) O.K. Kalashnikov, V.V. Klimov, Phys. Lett. B 88, 328 (1979);

(c) J. Kuti, J. Polonyi, K. Szalachani, Phys. Lett. B 98, 199 (1981);

(d) J. Rafelski, R. Hagedorn, From hadron gas to quark matter II, in Statistical Mechanics of Quarks and Hadrons, ed. by H. Satz (North Holland, Amsterdam, 1981)

7. V.V. Dixit, F. Karsch, H. Satz, Phys. Lett. B 101, 412 (1981)

8. (a) E.A. Guggenheim, J. Chem. Phys. 7, 103 (1939);

(b) I. Prigogine, Physica 16, 133 (1950);

(c) A. Münster, Statistical Thermodynamics (Springer, Berlin, 1969), Sects. 3.1 and 3.2;

(d) A. Münster, loc. cit.

9. A. Chodos, R.L. Jaffe, K. Johnson, C.B. Thorn, V.F. Weisskopf, Phys. Rev. D 9, 3471 (1974)

10. B. Touschek, Nuovo Cimento B 58, 295 (1968)

11. R. Hagedorn, I. Montvay, J. Rafelski, in Hadronic Matter at Extreme Energy Density, ed. by

N. Cabibbo, L. Sertorio (Plenum Press, New York, 1980)

12. R. Hagedorn, J. Rafelski, Commun. Math. Phys. 83, 563 (1982)

13. M.I. Gorenstein, V.K. Petrov, G.M. Zinovjev, Phys. Lett. B p106, 327 (1981) 